\title{
Effect of Semicon-Dielectric Interface on Conductivity and Electric Field Distribution
}

\author{
Hjerrild, J.; Holbøll, Joachim; Henriksen, Mogens; Boggs, S.
}

Published in:

IEEE Transactions on Dielectrics and Electrical Insulation

Link to article, DOI:

10.1109/TDEI.2002.1024438

Publication date:

2002

Document Version

Publisher's PDF, also known as Version of record

Link back to DTU Orbit

Citation (APA):

Hjerrild, J., Holbøll, J., Henriksen, M., \& Boggs, S. (2002). Effect of Semicon-Dielectric Interface on Conductivity and Electric Field Distribution. IEEE Transactions on Dielectrics and Electrical Insulation, 9(4), 596-603. https://doi.org/10.1109/TDEl.2002.1024438

\section{General rights}

Copyright and moral rights for the publications made accessible in the public portal are retained by the authors and/or other copyright owners and it is a condition of accessing publications that users recognise and abide by the legal requirements associated with these rights.

- Users may download and print one copy of any publication from the public portal for the purpose of private study or research.

- You may not further distribute the material or use it for any profit-making activity or commercial gain

- You may freely distribute the URL identifying the publication in the public portal 


\title{
Effect of Semicon-Dielectric Interface on Conductivity and Electric Field Distribution
}

\author{
Jesper Hjerrild, ${ }^{1}$ Joachim Holboll, Mogens Henriksen \\ Department of Electric Power Enginecring \\ Technical University of Denmark \\ DK-2800 Lyngby, Denmark \\ and Steven Boggs ${ }^{2}$ \\ Electrical Insulation Research Center \\ Departments of Electrical Engineering and Physics \\ University of Connecticut \\ Department of Electrical and Computer Enginecring \\ University of Toronto
}

\begin{abstract}
Development of solid dielectric dc transmission class cable has become a priority throughout much of the world. Interdiffusion between the semiconducting electrode materials and the dielectric inevitably causes variations in conductivity of the dielectric near the semicon which results in distortion of the electric field and space charge formation under de conditions. Analytical approximations and numerical computations provide a basis for analyzing space charge measurements, and based on such space charge measurements and the analysis, we estimate the field distortion for several material systems.
\end{abstract}

\section{INTRODUCTION}

$\mathrm{T}$ HE relative dielectric constant, $\epsilon_{r}$, of olefinic polymers is normally close to 2.3 and varies little with temperature. While the conductivity is a strong function of temperature and field, it never comes close to $\epsilon_{o} \epsilon_{r} \omega$ during normal operation, so that for ac-graded conditions, the cable always remains capacitively graded.

Under dc resistively graded conditions, the field grading depends on the conductivity of the dielectric, which can vary over orders of magnitude as a function of temperature and electric field. In the case of fluid-impregnated laminar dielectric cables, ion mobility in the fluid assures fairly uniform, although temperature-dependent, conductivity [1-7]. The nature of the laminar dielectric can be varied across the insulation to vary the conductivity as a function of radius. In the case of solid dielectrics, the thermal activation energy is generally quite large, in the range of 0.5 to $1 \mathrm{eV}$, which results in a very large temperature-dependence of the conductivity [8-10]. In addition, localized variations in conductivity can result from "contamination" of the dielectric from sources such as interdiffusion with semiconducting layers. Such interdiffusion and the effect thereof on dielectric properties has been documented in the literature [11].

INKT Research, Denmark

${ }^{2}$ Visiting Professor, Danish Technical University, July-October 2000.

Manuscript received on 5 January 2001, in final form 19 April 2002
In this contribution, we develop an analytic approximation for the space charge caused by such interdiffusion, provide more accurate numerical simulations of the space charge based on measured matcrial properties, and compare these simulations with actual measurements of space charge at the dielectric-semicon interface to estimate the variation of the conductivity.

As is well known, olefinic polymers have a band gap of about $8 \mathrm{eV}$ [8]; however, conduction is dominated by impurity states within the band gap, the nature of which is not really understood. However, any "contamination" of the polymer tends to increase the conductivity. The base polymer for carbon-filled semiconducting compounds is generally polar to ease the difficult task of dispersing the carbon black into the polymer. As a result, the chemical composition of semiconducting compounds differs substantially from that of the nonpolar compounds used as dielectrics, and diffusion of components from the semiconducting polymer into the dielectric constitutes "contamination" which is almost certain to increase the conductivity of the dielectric.

The ability to quantify, even indirectly, the effect of such interdiffusion-induced contamination on the local conductivity of the dielectric is of value in optimizing a dc cable system. An increased conductivity near the semicon will cause space charge formation and a reduction in the electric field under resistive grading, with a corresponding increase in the electric field after polarity reversal. 


\section{ANALYTIC APPROXIMATION}

Normal diffusion causes exponential concentration profiles, so that the most reasonable assumption is that the conductivity of the dielectric makes an exponential approach to its bulk value as a function of distance away from the semicon. With this assumption, the system is characterized by two parameters, the increase in conductivity at the semicon and the distance constant for approach to the bulk value.

In a resistively graded system, a variation in conductivity causes formation of space charge the magnitude of which is easily derived from Poisson's equation and the equation for current continuity as (for spatially invariant permittivity):

$$
\zeta(x)=J \epsilon \frac{d \rho(x)}{d x}
$$

where $\zeta$ is the space charge density, $\epsilon$ is the absolute dielectric constant, $J$ is the current density, and $\rho$ is the resistivity. As per above, we assume that the conductivity is given by

$$
\sigma(x)=\sigma(E, T)\left(1+\left(n_{\sigma}-1\right) \cdot \exp \left(\frac{-x}{\chi_{\sigma}}\right)\right)
$$

where the interface is at $x=0, n_{\sigma}$ is the factor by which the conductivity increases at the interface, and $\chi_{\sigma}$ is the distance constant which characterizes the decrease in conductivity to its bulk value.

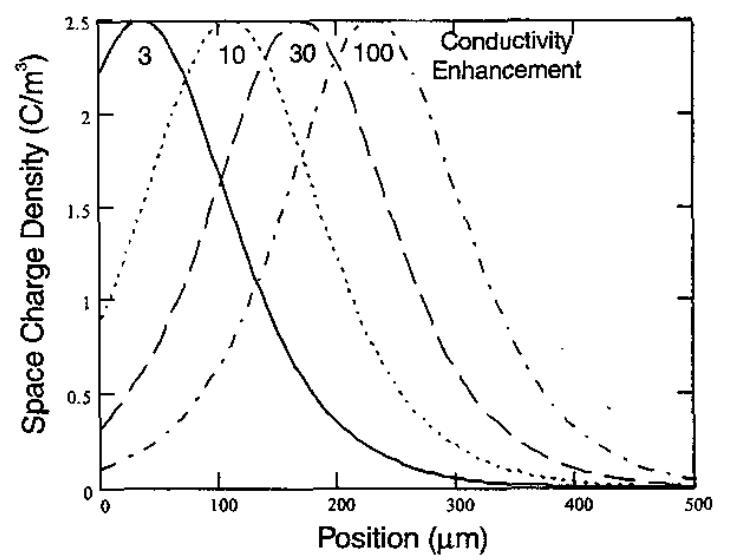

Figure 1. Space charge density computed from equation (3) with a distance constant, $\chi_{\sigma}$, of $50 \mu \mathrm{m}$, a bulk conductivity of $10^{-15} \mathrm{~S} / \mathrm{m}$, an average electric field of $25 \mathrm{kV} / \mathrm{mm}\left(J=25 \mathrm{nA} / \mathrm{m}^{2}\right)$, and relative dielectric constant of 2.3. Note that based on the assumptions of the analytic approximation, the peak magnitude of the space charge docs not change with the conductivity enhancement at the electrode, rather the position of the peak space charge shifts into the dielectric with increasing conductivity enhancement.

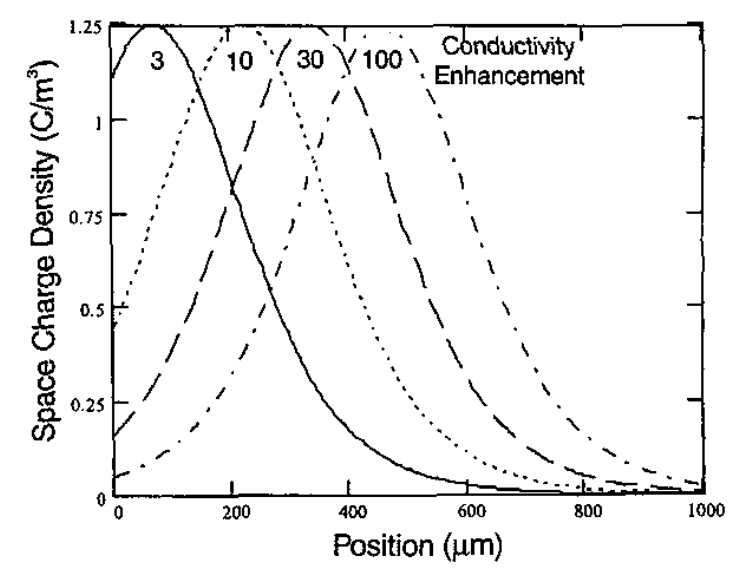

Figure 2. Similar conditions to Figure 1 except that the distance constant has been increased to $100 \mu \mathrm{m}$; note the change in position scale. The position of the peaks has increased by a factor of two; however, the pcak space charge has decreased by a factor of two.

If we invert equation (2) to obtain the resistivity and substitute into equation (1), we obtain for the space charge,

$$
\zeta(x)=\frac{J \epsilon}{\sigma(E, T) \chi_{\sigma}} \frac{\left(n_{\sigma}-1\right) \exp \left(\frac{-\chi}{\chi_{\sigma}}\right)}{\left(1+\left(n_{\sigma}-1\right) \exp \left(\frac{-\chi}{\chi_{\sigma}}\right)\right)^{2}}
$$

Figures 1 and 2 show the space charge plotted as a function of conductivity enhancement at the semicon for different distance constants $\chi_{\sigma}$. Based on this analysis, we find that space charge generated by variation in polymer conductivity near the electrode is Gaussian-like, varies little in magnitude with conductivity enhancement, but varies substantially in position of the peak relative to the electrode with conductivity enhancement.

We can also evaluate the field distortion caused by the variation in conductivity. Since the distorted region is very small relative to the $9 \mathrm{~mm}$ thickness of the dielectric, it has little effect on the current density. Thus assuming the current density is determined by the bulk material (here assumed to have a conductivity of $10^{-15} \mathrm{~S} / \mathrm{m}$ ), the current density is just the product of the bulk conductivity and the field, which we will assume to be $20 \mathrm{kV} / \mathrm{mm}$ to give a current density of $20 \mathrm{nA} / \mathrm{m}^{2}$. The electric field is then just the current density divided by the conductivity and is shown in Figure 3. As expected, substantial enhancements of the conductivity bring the field near the electrode close to zero. This might be seen as an advantage in that it shields defects at the electrode from the field. The problem, of course, the drop in field for the resistively graded condition becomes an increase in field at the electrode upon polarity reversal. Thus a relatively small increase in conductivity near the electrode can cause a very substan- 


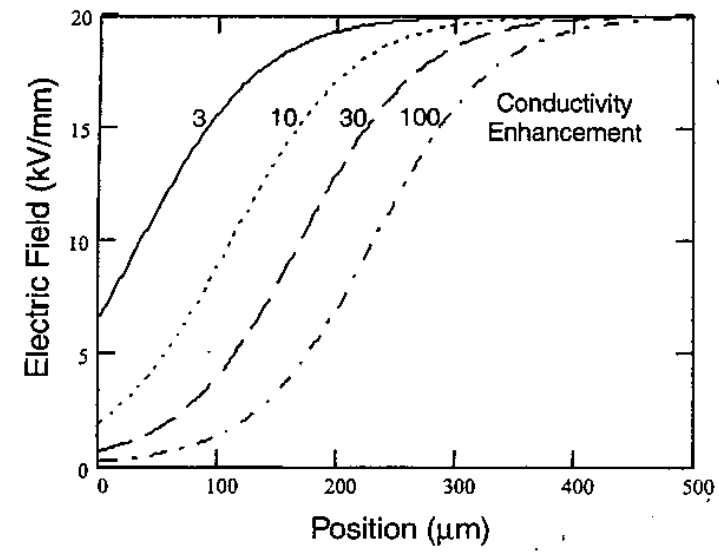

Figure 3. Analytic approximation to the resistively graded electric field distribution near the electrode as a function of conductivity enhancement.

tial increase in the field at the electrode upon polarity reversal, and a factor of 10 is a "small" change in conductivity as variations of 5 or 6 orders of magnitude are possible as a function of temperature and field.

\section{NUMERICAL SIMULATIONS}

The above analytical approximation does nòt take into account the effect of field-dependent conductivity, which can be appreciable above $10 \mathrm{kV} / \mathrm{mm}$. Numerical simulations were therefore undertaken for a range of materials including one with a very large field coefficient of conductivity $\left(\sigma_{1}\right)$ and one with a more typical field coefficient $\left(\sigma_{2}\right)$ (equations (4 and 5), respectively and Figure 4). The coefficients in these equations were derived from fits to measured data over the temperature range from 40 to $80^{\circ} \mathrm{C}$ and at fields from 10 to $30 \mathrm{kV} / \mathrm{mm}$. All simulations were undertaken at a temperature of $25^{\circ} \mathrm{C}$. The means by which the field coefficient was measured have been described in [12].

$$
\begin{array}{r}
\sigma_{1}(E, T)=3.2781 \exp \left(\frac{-0.56 \cdot q}{k_{B} T}\right) \\
\times \frac{\sinh \left(2.7756 \times 10^{-7}|E|\right)}{x^{\prime}|E|} \\
\sigma_{2}(E, T)=3.6782 \times 10^{7} \exp \left(\frac{-0.98 \cdot q}{k_{B} T}\right) \\
\times \frac{\sinh \left(1.086 \times 10^{-7}|E|\right)}{|E|}
\end{array}
$$

The numerical simulations employ a custom program for transient nonlinear finite element analysis which iterates to the self-consistent solution for the electric potential, given that the field depends on the conductivity and the conductivity is a function of the field [13]. The output from the program is the electric field as a function of po-

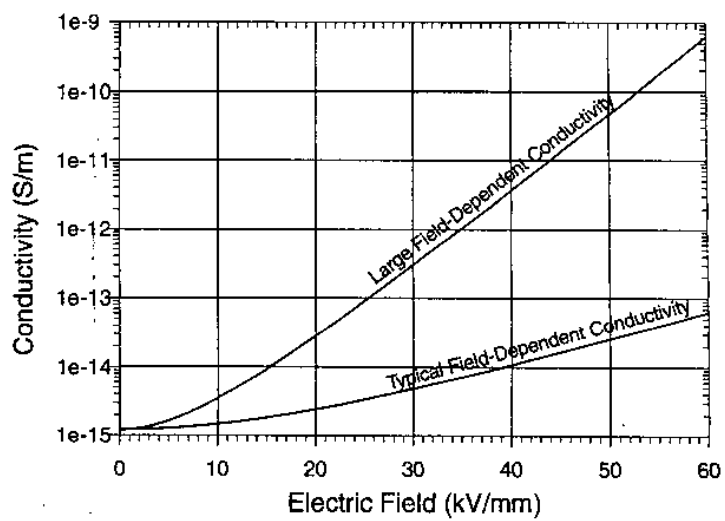

Figure 4. Conductivity at about $45^{\circ} \mathrm{C}$ as a function of electric field for the $\sigma_{1}$ and $\sigma_{2}$ as provided in equations ( 4 and 5 ).

sition in the dielectric, from which we can compute the conductivity as a function of position from an equation such as 4 or 5 , which is an "input" to the program. The electric field will be qualitatively similar to Figure 3 . However, the electric field as provided by the program is not very smooth as it must be computed from the derivative of the potential. In order to proceed with the analysis, we need to take a derivative of the resistivity with respect to position, which is essentially equivalent to taking a derivative of the field. To facilitate this, we fit an analytic function to the field as a function of position.

The function

$$
E(x)=y_{0}+\frac{a}{\left(1+\exp \left(\frac{x_{o}-x}{b}\right)\right)^{c}}
$$

where $a, b, c, x_{o}$, and $y_{0}$ are constants, fits the numerically computed data (dots in Figure 5) extremely well. We can substitute equation (6) into equation (5), invert the result to obtain the resistivity, take a derivative with re-

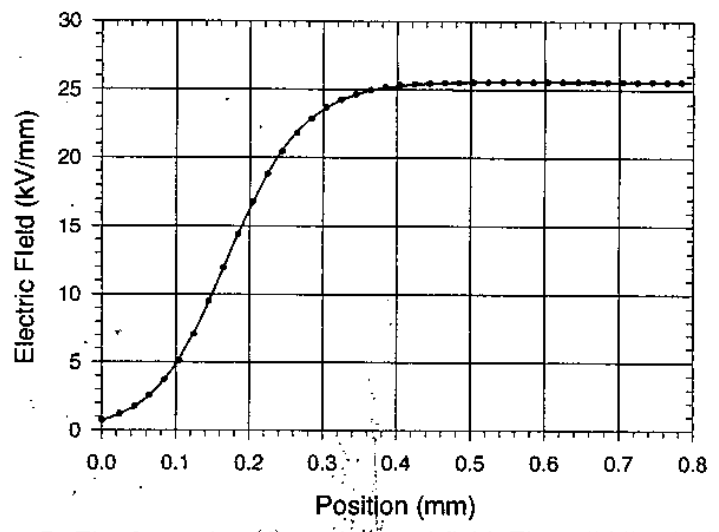

Figure 5. Fit of equation (6) to computed field. The solid line is the fit, and the dots are every fifth datum in the computed field. The data are computed for a conductivity corresponding to equation (5) with an enhancement factor of 100 at the electrode. This is a near worst case fit, as the goodness of fit decreases with increasing enhancement factor. The average field is $20 \mathrm{kV} / \mathrm{mm}$; however, the large reduction in field at the electrodes causes the field in the bulk to rise to about $25 \mathrm{kV} / \mathrm{mm}$. 


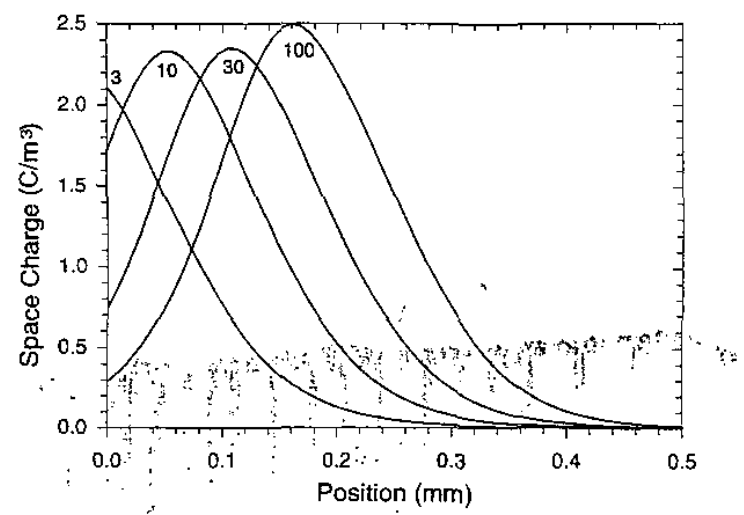

Figure 6. Numerically computed space charge density (including the effect of field-dependent conductivity) as a function of position for the material characterized by equation (5) with factors of $3,10,30$ and 100 increase in conductivity at the electrode and a distance constant of $50 \mu \mathrm{m}$.

spect to $x$, multiply by $J \epsilon$, and obtain an equation for the space charge density as a function of position. Although the equation is much too long to print, computed data are shown in Figure 6 for the field distribution of Figure 5.

In comparing Figure 6 with Figure 1, we see good quantitative agreement in the space charge magnitude but substantial differences in the position and shape of the space charge distribution. For example, the numerical data for 100 times conductivity enhancement look more like the analytical approximation for 30 times rather than 100 times. This is undoubtedly the result of the field-dependent conductivity of the dielectric. From Figure 4, we can see that the conductivity for a field of $20 \mathrm{kV} / \mathrm{mm}$ is about 5 times that for no field. According to Figure 5, the field near the electrode is nearly zero, so that the conductivity will vary substantially within the problem, and since the space charge is a direct function of the spatial variation of the conductivity, we cannot expect quantitative agreement between the analytic approximation and the more accu-

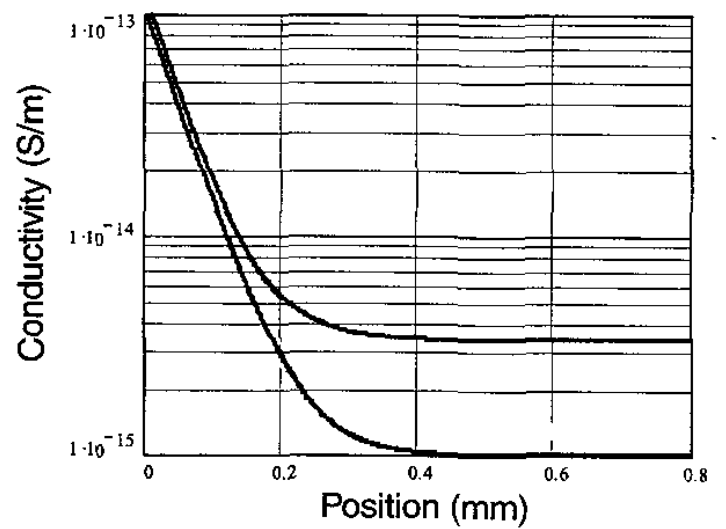

Figure 7. Conductivity as a function of position for the conditions of Figure 6. The lower curve shows the conductivity without the effect of field-dependent conductivity, while the upper curve shows the actual conductivity in a dielectric characterized by equation (5), including the effect of field-dependent conductivity.

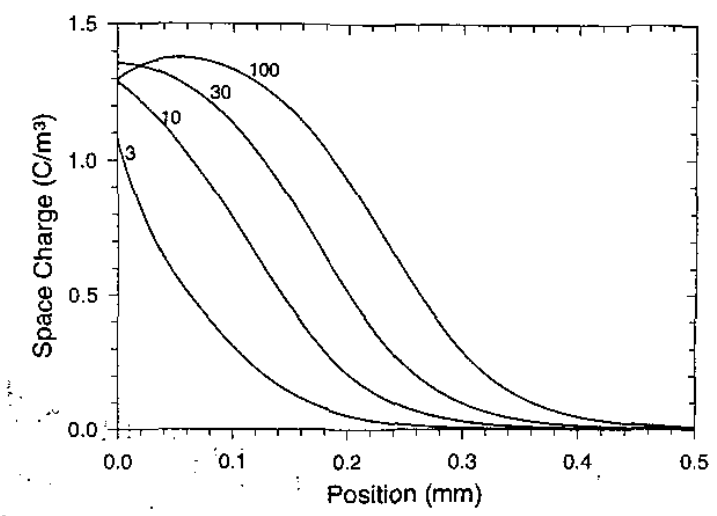

Figure 8. Numerically computed space charge density (including the effect of field-dependent conductivity) as a function of position for the material characterized by equation (4) with factors of $3,10,30$ and 100 increase in conductivity at the electrode and a distance constant of $50 \mu \mathrm{m}$. The conductivity of this material changes much more rapidly with field than "normal" polymers with the result that the space charge distribution at the electrode is highly "distorted".

rate numerical simulations. Indeed, if we compute the conductivity as a function of position based on the data of Figure 5, we arrive at Figure 7, which indicates that although the conductivity of the polymer increased by a factor of 100 at the electrodes, the actual variation in the conductivity between the bulk matcrial (at $25 \mathrm{kV} / \mathrm{mm}$ ) and the electrode (near zero field) is only a factor of 30 as a result of the field-induced increase in conductivity in the bulk. This explains why the numerically computed curve is much closer to the analytical curve for an enhancement factor of 30 than to that for 100 , even though the computation which resulted in Figure 6 was for a conductivity enhancement factor of 100 at the electrodes, i.e., was of the form of equation (2), where $\sigma_{2}(E, T)$, i.e., equation (5), was substituted for $\sigma_{0}$. We also note that the amplitude of the space charge is not constant in Figure 6 and approaches that of the analytical approximation with increasing conductivity enhancement.

Figure 8 shows the results of numerical simulations on a material with the field-dependent conductivity characteristics corresponding to equation (4). This material has a much greater field-dependent conductivity than a "normal" polymer and results in extreme distortion of the space charge relative to the analytic approximation or the more typical material characterized in Figure 6.

After examining Figures 6 and 8, we can see very clearly that quantitative interpretation of space charge measurements in the present context (and in many contexts) requires knowledge of the field-dependent (and if temperature gradients are involved, temperature-dependent) conductivity followed by computations which include these phenomena. The effects of field-dependent material properties under dc conditions, even at normal engineering fields, are not second order effects. They are often the dominant effect. 


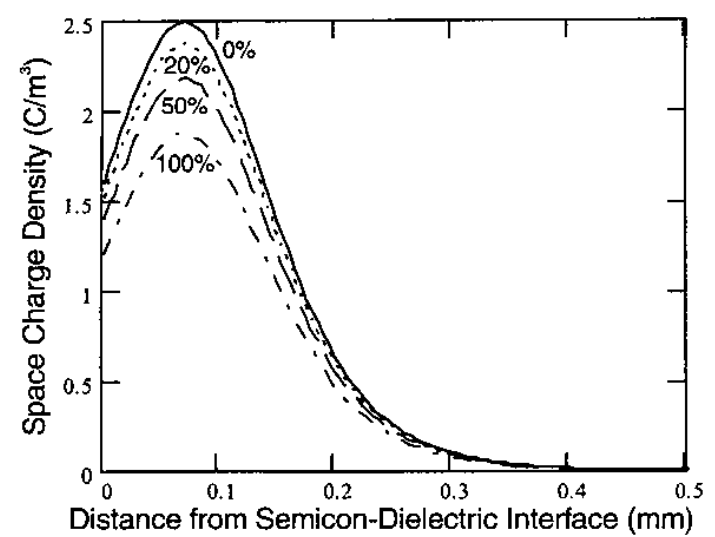

Figure 9. Analytical approximation for the effect of permittivity variation near the semicon-dielectric interface on the space charge under resistively graded conditions. The dielectric constant of the bulk is assumed to be 2.25 with percent enhancements at the semicon-dielectric interfacc as shown in the figure. The distance constant for the exponential drop in dielectric constant and conductivity to bulk values is $50 \mu \mathrm{m}$ with a conductivity enhancement factor of 5 .

\subsection{EFFECT OF PERMITTIVITY}

Equation 1 is derived from Poisson's equation with the assumption that the permittivity is not a function of position. When this assumption is relaxed, equation (1) becomes

$$
\xi(x)=J\left(\epsilon \frac{d \rho(x)}{d x}+\rho \frac{d \epsilon(x)}{d x}\right)
$$

If both the conductivity and permittivity increase in the dielectric as the semicon is approached, then the first term in equation (7) will be negative while the second term is positive. Thus the effect of the spatial variation in the permittivity is to decrease the space charge density near the semicon. Of course, if the permittivity were decreasing as the semicon is approached, the effect would be to increase the space charge. This could happen if, for example, highly polar volatile cross linking byproducts, such as acetophenone, were diffusing out of the dielectric. The semicon is probably much more permeable than the dielectric, so that diffusion would cause a density gradient at the interface which could result in a decreasing dielectric constant as the semicon is approached.

Figure 9 shows a computation in which the dielectric constant has been modeled as

$$
\epsilon(x)=\epsilon_{o} \epsilon_{r}\left(1+\left(n_{\epsilon}-1\right) \cdot \exp \left(\frac{-x}{\chi_{\epsilon}}\right)\right)
$$

while the conductivity is modeled as per equation (2). The distance constants, $\chi_{\sigma}$ and $\chi_{\epsilon}$, are $50 \mu \mathrm{m}$ for the permittivity, and conductivity, and the conductivity enhancement factor, $n_{\sigma}$, is 5 .

As noted above and seen in Figures 6 and 8, the peak in the space charge distribution moves into the bulk of the dielectric with increasing conductivity enhancement factor because the space charge density depends on the derivative of the resistivity rather than conductivity. However, the effect of the dielectric constant depends on the derivative thereof which is maximum at the electrode. Thus if we had plotted Figure 9 for a conductivity enhancement factor of 50 , the variation in dielectric constant would have negligible effect on the space charge distribution, as the peak in the space charge distribution would have been well into the dielectric, where the variation in dielectric constant is small.

\section{SEMICON-INDUCED CONDUCTIVITY}

\subsection{SPACE CHARGE MEASUREMENT}

For the above reasons, we have measured the fielddependent conductivity of several polymers along with the field-induced space charge at the semicon-dielectric interface. In the case of cable samples, the space charge formation over time was measured using the laser induced pressure pulse method. The geometric field near the inner semiconductor was $20 \mathrm{kV} / \mathrm{mm}$ and the laser pulse energy was $450 \mathrm{~mJ}$ with a pulse width of $7 \mathrm{~ns}$. The laser pulse was applied directly to the surface of the ground shield semiconductor. The space charge induced signal was measured using a coupling capacitor of $1.5 \mu \mathrm{F}$ to an amplifier with a $20 \mathrm{MHz}$ bandwidth. In the case of plaque samples, space charge was measured using the pulsed electroacoustic method. The geometric field across the dielectric was again $20 \mathrm{kV} / \mathrm{mm}$. The amplitude of the electric pulse was $2 \mathrm{kV}$ with a pulse width of $25 \mathrm{~ns}$. The space charge induced signal was recorded using a digital oscilloscope.

\subsection{CONDUCTIVITY AT THE SEMICON-DIELECTRIC INTERFACE}

Knowledge of the field-dependent conductivity and field-induced space charge at the semicon-dielectric interface provides a basis for evaluating the field-dependent conductivity and field distortion at the electrode. Figure 10 shows the measured space charge near the semicon-dielectric interface along with several numerically computed fits for the less conductive material (equation (5)). The large peak to the left is the induced charge on the electrode caused by the applied voltage. The conductivity-induced space charge appears to be distorted in the immediate vicinity of the electrode, probably by a blocking effect at the electrode. Three computed curves have been plotted for comparison with the measured data. The one corresponding to $100 \mathrm{x}$ conductivity enhancement and 100 $\mu \mathrm{m}$ distance constant is clearly not a good fit. The other two curves which correspond to conductivity enhancements of 1000 and 500 and distance constants of 100 and $120 \mu \mathrm{m}$, respectively, are better fits to the data. As noted above, the distance constant determines the magnitude of 


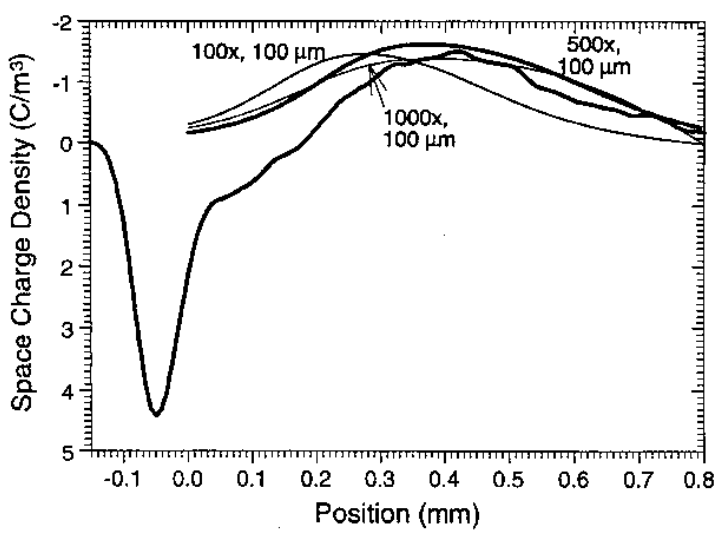

Figure 10. Measured space charge along with several numerically computed fits for the less conductive material (equation (5)). The large peak to the left is the induced charge on the electrode caused by the applied voltage. The conductivity-induced space charge appears to be distorted in the immediate vicinity of the electrode, probably by a blocking effect at the electrode. Three computed curves have been plotted for comparison with the measured data. The one corresponding to $100 \mathrm{x}$ conductivity enhancement and $100 \mu \mathrm{m}$ distance constant is clearly not a good fit. The other two curves which correspond to conductivity enhancements of 1000 and 500 and distance constants of 100 and $120 \mu \mathrm{m}$, respectively, are better fits to the data.

the space charge, so that a distance constant in the range of $100 \mu \mathrm{m}$ is necessary to fit the measured space charge magnitude. The position of the space charge peak is determined primarily by the conductivity enhancement, and a conductivity enhancement in the range of 500 to 1000 is required to obtain a reasonable match with the measured peak position. Thus the data suggest that the conductivity near the semicon-dielectric interface is enhanced in the range of 1000 and falls off with a distance constant in the range of $100 \mu \mathrm{m}$. Such a change in conductivity near the electrode would be of no consequence under ac-graded conditions but has a major effect on the field distribution under resistively graded conditions.

Figure 11 shows similar data for the more conductive material (equation (4)). The primary difference in this material is that the space charge disappears almost immediately when the voltage is removed. This tends to suggest that the space charge is caused by the current density according to equation (1) or, more generally, equation (7). According to equation (4), this material has a conductivity of about $8.2 \mathrm{E}-15 \mathrm{~S} / \mathrm{m}$ at $300 \mathrm{~K}$ and $20 \mathrm{kV} / \mathrm{mm}$, the conditions of the space charge measurements. This results in a dielectric time constant of about $2400 \mathrm{~s}$. However, if the conductivity near the electrode were enhanced by a factor of 500 , the dielectric time constant would be in the range of $5 \mathrm{~s}$, and the space charge would disappear too rapidly to be observed after the voltage is removed. Thus the observation that the space charge in this sample disappears before it can be observed after the voltage is removed (within about $100 \mathrm{~s}$ ) tends to confirm that the conductivity in the dielectric near the electrode is enhanced substantially.

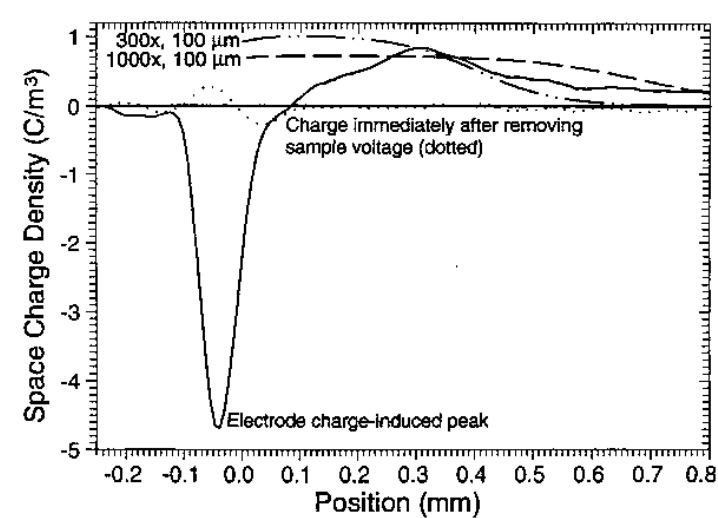

Figure 11. Space charge data and fits thereto for the more conductive material (equation (4)). The dotted line shows a space charge measurement immediately after the voltage was removed. In this material, the space charge disappears almost immediately when the voltage is removed, which tends to indicate that the space charge is caused by the current density passing through an inhomogeneous conductivity. Again, the magnitude of the space charge peak pretty well dictates that the distance constant must be about $100 \mu \mathrm{m}$. Computed curves with this distance constant are shown for conductivity enhancements of 300 and 1000 . The latter appears to fit better, but in any case, the conductivity enhancement must be in this range if the space charge is caused thereby.

Again, the distance constant must be in the range of $100 \mu \mathrm{m}$ to obtain the appropriate space charge amplitude. The enhancement factor is more difficult to judge in this highly nonlinear material, as the strong field-dependence of the conductivity distorts the space charge distribution as seen in Figure 8. Computed data for conductivity enhancements of 300 and 1000 are shown, and the latter appears to be the better fit. Thus in both materials, the conductivity appears to be enhanced in the range of 500 to 1000 near the semiconducting electrodes.

\subsection{IMPLICATIONS FOR DC CABLE}

The electric field distribution was solved for a cable with $18 \mathrm{~mm}$ radius conductor and $9 \mathrm{~mm}$ thick dielectric with field and temperature-dependent conductivity characterized by either equation (4) or equation (5), $150 \mathrm{kV}_{\mathrm{dc}}$ applied, a conductor temperature of $85^{\circ} \mathrm{C}$ and a ground temperature of $45^{\circ} \mathrm{C}$. As stated earlier, the equations for conductivity as a function of electric field and temperature are based on measurements of commercially available polymers. The conductivity of the dielectric at each semicon was assumed to increase by a factor of 1000 with a distance constant of $100 \mu \mathrm{m}$. Figure 12 shows the resistively graded electric field and the field immediately after a polarity reversal for such cables. As a result of the large conductivity enhancement at the electrodes, the field at the electrodes is near zero under resistive grading. This might be seen as an advantage for the resistively graded condition; however, upon polarity reversal, this reduction turns into an enhancement which results in a field of over $40 \mathrm{kV} / \mathrm{mm}$ at the conductor while the average field is only $16.67 \mathrm{kV} / \mathrm{mm}$, as seen in Figure 12 . Without the conduc- 


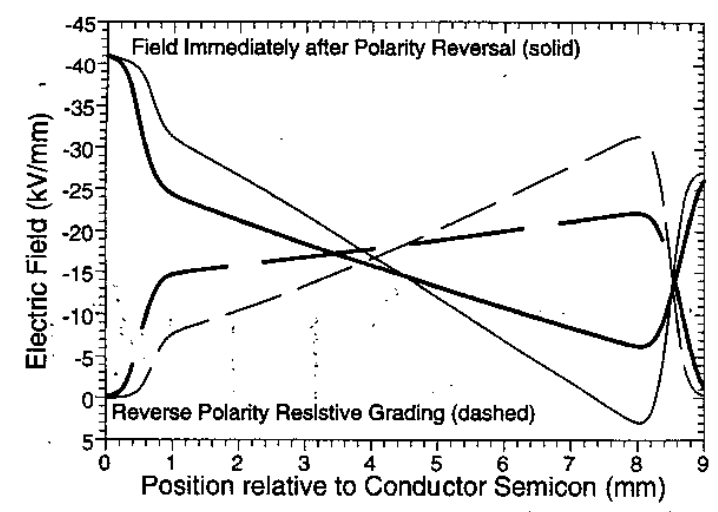

Figure 12. Resistively graded field distribution (dashed lines) for the material characterized by equation (4) (heavy lines) and equation (5) (light lines) along with the field distribution immediately after a polarity reversal (solid lines). The conductivity-induced reduction in the field at the semicons might be considered an advantage under resistive grading; however it results in a very large, "transient" field under polarity reversal. The duration of the transient depends on the material and temperature, and could vary from minutes to many hours. The applied voltage is $150 \mathrm{kV}$ across $9 \mathrm{~mm}$ for an average field of $16.67 \mathrm{kV} / \mathrm{mm}$. Upon polarity reversal, the field at the conductor reaches over $40 \mathrm{kV} / \mathrm{mm}$ before relaxing back to nearly zero. Without the conductivity enhancement at the conductor, the maximum field upon polarity reversal would be $28 \mathrm{kV} / \mathrm{mm}$ and $37 \mathrm{kV} / \mathrm{mm}$ for the materials characterized by equation (4) and equation (5), respectively.

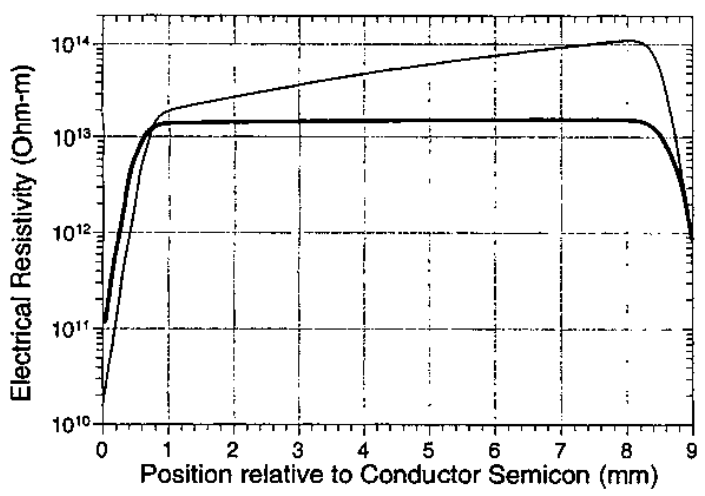

Figure 13. Insulation resistivities for the resistively graded cable dielectrics characterized by equation (4) (heavy line) and equation (5) (light line) with a conductivity enhancement of 1000 at each semicon with a distance constant of $100 \mu \mathrm{m}$. The applied voltage is $150 \mathrm{kV}$ across $9 \mathrm{~mm}$. The conductor is at $85^{\circ} \mathrm{C}$ while the ground is at $45^{\circ} \mathrm{C}$ The very large field-dependent conductivity combined with the low thermal activation energy of the "heavy line" material results in almost uniform resistivity through the bulk of the material in spite of the large temperature gradient. Since the space charge is proportional to the spatial derivative of the resistivity, little space charge is created in the bulk of the dielectric. For the material with greater activation energy and less field-dependent conductivity (light line), the much greater variation in resistivity across the dielectric results in a small space charge in the bulk as seen in Figure 14. However, the really large effect is at the electrodes where the conductivity varies by several orders of magnitude over a small distance. However even here, the large field-dependent conductivity of the heavy line material (equation (4)) causes the resistivity to vary by only 1 to 2 orders of magnitude near the electrode rather than the 2 to 3 orders of magnitude for the light line material (equation (5)).

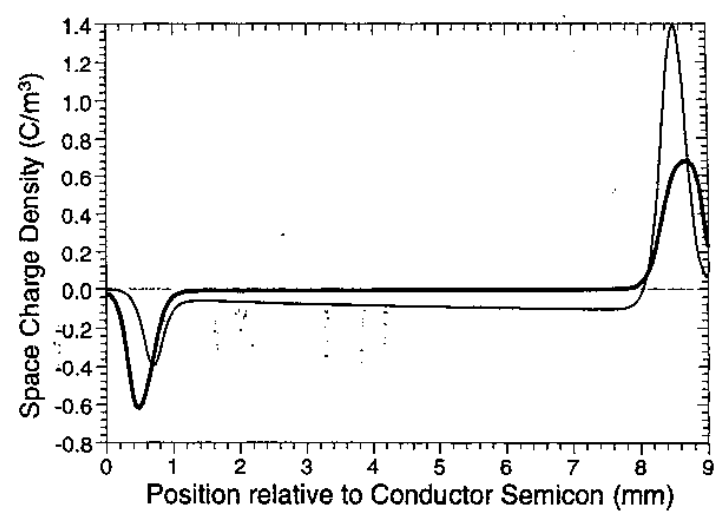

Figure 14. Space charge distribution for the resistively graded cable dielectrics characterized by equation (4) (heavy line) and equation (5) (light line) with a conductivity enhancement of 1000 at each semicon with a distance constant of $100 \mu \mathrm{m}$. The applied voltage is $150 \mathrm{kV}$ across $9 \mathrm{~mm}$. The conductor is' at $85^{\circ} \mathrm{C}$ while the ground is at $45^{\circ} \mathrm{C}$, which results in differing fields, resistivities (Figure 13), and space charge distributions in the vicinity of the two electrodes (at 0 and 9 $\mathrm{mm})$.

tivity enhancement at the semicons, the maximum fields upon polarity reversal would be $28 \mathrm{kV} / \mathrm{mm}$ for the equation (4) material and $37 \mathrm{kV} / \mathrm{mm}$ for the equation (5) material based on similar numerical simulations. The high field in the case of the equation (5) material results from the large thermal activation energy of the conductivity $(0.98$ $\mathrm{eV}$ ) and the low field-dependence, which combine to cause - the conductivity to vary substantially across the conductor [12]. The much lower thermal activation energy of the equation (4) material $(0.56 \mathrm{eV})$ and much greater field-dependence result in a much smaller variation in the conductivity across the dielectric. We note that under capacitive grading, the maximum field at the conductor would be $20.5 \mathrm{kV} / \mathrm{mm}$ for $150 \mathrm{kV}$ applied voltage.

The large variation in resistivity (Figure 13) at the electrodes results in substantial space charge as seen in Figure 14. The variation in resistivity both in the bulk and near the cable semicons is much smaller in the material of equation (4) than that of equation (5) for the reasons explained above with the result that the space charge both in the bulk and at the semicons is lower in the cable made with the equation (4) material than the equation (5) material.

\section{CONCLUSIONS}

$\mathrm{M}$ EASUREMENTS of space charge adjacent to semiconducting electrodes can be explained by assuming a field-dependent conductivity near the semicon caused by interdiffusion between the semicon and the dielectric. Such interdiffusion is known to occur and to affect dielectric properties [11]. Analysis of such data from two very different dielectrics with the same semiconducting electrodes suggests a conductivity enhancement in the 
range of 1000 at the electrodes with a distance constant of about $100 \mu \mathrm{m}$. Such a conductivity variation will increase the maximum field upon polarity reversal by in the range of $50 \%$ for a "good" dc cable dielectric, although it will have little effect on the maximum field under polarity reversal for a "poor" dc cable dielectric. In this context, a good dc cable dielectric has a small thermal activation energy of conductivity and a large field-dependent conductivity [12]. Numerical simulations and analytic approximátions provide a basis for evaluating space charge measurements to infer the variation in conductivity at a semicondielectric interface:

\section{REFERENCES}

[1] G. Bahder, F. Garcia and A.S. Brookes, "Insulation Coordination in High Voltage DC Cables", CIGRE Paper 21-03, 1972.

[2] G. Bahder, G. S. Eager, G. W. Seman, F. E. Fischer, and H. Chu, "Development of $\pm 400 \mathrm{kV} / \pm 600 \mathrm{kV}$ High and MediumPressure Oil-filled Paper Insulated DC Power Cable System", IEEE Trans. PAS-97, pp. 2045-2056, 1978.

[3] A. L. McKean, and E.M. Allam, "Progress in Development of $\pm 600 \mathrm{kV}$ DC Cable System", IEEE Trans. PAS-99, pp. $1251-1258,1980$.

[4] E. M. Allam and A. L. McKean, "Design of an Optimized \pm 600 kV DC Cable System", IEEE Trans. PAS-99, pp. 1713-1721, 1980.

[5] C. A. Arkell and A. F. Parsons, "Insulation Design of Self-Contained Oil-Filled Cables for DC Operation", IEEE Trans. PAS. 101, pp. $1805-1814,1982$
[6] O. Haugc, J. N. Johnsen, T. A. Holte, and K. Bjorlow-Larsen, "Performance of the $+250 \mathrm{kV}$ HVDC Skagerrak Submarine Cables, Further Development of the HVDC Paper-Insulated, Mass-Impregnated (Solid Type) Submarine Cable", IEEE Trans. PD-3, pp. 1-15, 1988.

[7] F. G. Garcia, "Design Procedures for High-Voltage, Direct Current Transmission Cables", IEEE Trans. PD-3, pp. 425-433, 1988.

[8] A. R. Blythe, Electrical Properties of Polymers, Cambridge University Press, 1986.

[9] H. Fukágawa; H. Miyauchi, Y. Yamada, S. Yoshida, and N. Ando, "Insulation Properties of 250 kV: DC XLPE Cables", IEEE Trans: PAS 100, pp. 3175-3184, 1981.

[10] B. M.Weedy and D. Chu, "HVDC Extruded Cables - Parameters for Determination of Stresses", IEEE Trans. PAS-103, pp. $662-667,1984$.

[11] J. Bezille, H. Janah, J. Chan, and M. D. Hartley, "Influence of Diffusion on Some Electrical Properties of Synthetic Cables", IEEE Conference on Electrical Insulation and Dielectric Phenomena, pp. 567-572, 1992

[12] S. A. Boggs, J. Hjerrild, J. Holboll, and M. Henriksen, "Effect of Insulation Properties on the Field Grading of Solid Dielectric DC Cable", Submitted for publication.

[13] J. Kuang, J. D. Lavers, and S. A. Boggs. "Program for Transient Nonlinear Finite Element Analysis with Applications to Coupled Field Problems", Proceedings ISH (International Symposium on High Voltage Engineering), Montreal, Canada, Vol. 6, pp. 141-144, 1997. 